\title{
Future Directions in Natural Language Processing Applications in Smoking Cessation Therapy
}

\section{PROKOP, J.}

Czech Technical University, Faculty of Electrical Engineering, Department of Cybernetics, Prague, Czech Republic
Citation | Prokop, J. (2021). Future directions in natural language processing applications in smoking cessation therapy. Adiktologie, 21(1), 51-57. https://doi.org/10.35198/01-2021-001-0003
INTRODUCTION: Behavioural support has been shown to possess high efficacy in aiding smokers to stop smoking at a level at least similar to nicotine replacement therapies (NRT) while causing no adverse physiological effects. Furthermore, the effects of behavioural interventions and NRT appear to be roughly additive. The effectiveness of interventions appears to increase with the frequency of contact with an eHealth application, with daily contact showing higher efficacy compared to weekly sessions with a trained stopsmoking specialist. Previous automated counsellors based on a motivational interview approach, lapse preparation, and lapse management are rigid in their therapeutic path, with limited ability to reflect the needs and the specific situation of a given patient. AlMS: This report aims to describe a possible approach to developing a more engaging, patient-tailored automated counsellor based on recent advances in the natural language processing (NLP) field that should make remote chat-based counselling easier for professionals while gathering data for the NLP model, which should ultimately be able to conduct the therapy on its own. METHODS: The core of the approach lies in utilizing the Text-to-text transfer transformer (T5). T5 is, in essence, a set of neural network models aimed at tasks formulated as an expected textual response to a given textual input. These models can be utilized to - at first - suggest answers to counsellors in live chat sessions with patients. Actual answers from these sessions would subsequently be used to fine-tune the models and ultimately provide high-quality counselling without human intervention on the therapist's side. CONCLUSION: The article presents a novel approach to internet-delivered smoking cessation cognitivebehavioural therapy utilizing a powerful artificial neural network NLP model acting as a conversational agent and a data collection protocol with usage incentives for both smoking cessation experts and smokers.

\section{Keywords $\mid$ eHealth - Smoking Cessation - Chatbot - Online Intervention - Natural Language Processing -}

\section{Transfer Learning}




\section{INTRODUCTION}

Worldwide, an estimated $23 \%$ of the adult (at least 15 years old) population, or approximately a billion people, smoke tobacco (Gowing et al., 2015), causing approximately six million deaths every year (West, 2017). According to Jha \& Peto (2014), most smokers start smoking in their youth (50\% of men, 10\% of women), they rarely stop, their life expectancy is, on average, ten years lower when compared to that of non-smokers, and they can expect the onset of an age-related disease about ten years earlier compared to non-smokers. However, the same source reports that stopping smoking before 30 years of age can reverse the effects entirely, and stopping later has a significant positive impact on life expectancy - stopping at 50 years of age still increases a person's life expectancy by six years on average.

Smoking cessation is notoriously hard; according to West (2017), only about 5\% of unaided attempts to stop smoking are successful in the long term (no relapse for at least six months). The same article cites various forms of behavioural support to increase the odds: addiction expert counselling by 2-7 percentage points, printed self-help materials by 1-2 percentage points, text messaging programmes by 2-7 percentage points. According to Kulhánek et al. (2018), the long-term success rate of quit attempts aided by continuous everyday behavioural therapy in the form of a specific eHealth application is at least $20 \%$, which is significantly higher than the $7-12 \%$ success rate of the more efficient methods mentioned above. Furthermore, the same study estimates that roughly three-quarters of smokers would prefer quitting with the aid of an eHealth application instead of attending sessions with an addiction expert.

Given the above, effective and scalable tools that aid smoking cessation are highly desirable. Cognitive behavioural therapy approaches are effective, but conventional therapy is not scalable to the figures mentioned above. This work proposes an artificial neural network-based agent based on recent advances in the natural language processing field and a protocol for collecting the data needed to train it.

\subsection{Natural language processing applications in public health}

Natural language processing (NLP) has been successfully applied to many issues in the health sciences. NLP is tackling a rapidly increasing number of various tasks. A considerable portion of previous successful applications focused on the processing of unstructured medical text (e.g. anamneses or discharge summaries). The benefit of these applications arises chiefly from the following facts. One, a typical medical record is too big for a person to absorb in a limited time. Two, there are many tasks and research questions in the medical field whose solution requires the valuable information contained within the medical records in a specific form. Three, up to $70 \%$ of a typical medical record is an unstructured text (Roberts, 2017). Two notable examples are information extraction and text summarization. A comprehensive review of clinical information extraction applications is given by Wang et al. (2018). Other examples are prediction, e.g. symptom severity prediction from neuropsychiatric records (Filannino et al., 2017) or problem usage of prescription drugs (Carrell et al., 2015), classification, e.g. classification of a cancer stage from free-text histology reports (McCowan et al., 2006), analysing social media for public sentiment, prevalence, and the like with regard to medical topics (Conway et al., 2019), and, importantly, de-identification. With the exception of de-identification, all these tasks fall into a subfield of natural language understanding (NLU). A comprehensive summary of such NLP applications in the clinical field is given by Velupillai et al. (2018). Many of these applications are already well established, with several ready-to-use software frameworks, e.g. SemEHR (Wu et al., 2020), cTAKES (Savova et al., 2010), and codified benchmark tasks such as n $2 \mathrm{c} 2 .^{1}$

The above NLP applications in the medical field may be regarded as "passive". That is, their ultimate use is to inform medical professionals who then implement treatments. In contrast, recent advances in NLP allow the treatment itself to be tackled by automated systems. Specifically, conversational agents (chatbots) in mental health therapies have started emerging. An overview of 41 different mental health support chatbots is given by Abd-alrazaq et al. (2019). Out of these, only four were based on artificial intelligence (a more detailed description was not provided), and the rest were rule-based systems. The artificial intelligence approach, or, more specifically, the artificial neural network approach and the rule-based approach, differs substantially from the technical NLP perspective, from the perspective of clinical experts creating and managing such systems, and in their capabilities.

\subsection{Chatbots in smoking cessation}

Currently, chatbots for the support of smoking cessation are almost non-existent. A relatively complex embodied conversational agent for assessing tobacco and alcohol abuse was described by Auriacombe et al. (2018). Despite the overall complexity of the solution, this system merely asks a predefined set of questions and extracts the answers. A notable effort towards active support for quitting smoking is presented in Calvaresi et al. (2019). This system's conversational agent relies on a state machine. The lack of existing neural conversational agents in the field is primarily caused by the relative novelty of reasonably-performing neural chatbots themselves.

\section{METHODS}

\subsection{Artificial neural networks}

Machine learning techniques, by definition, overcome the difficulty of fully specifying how a computer program should behave and instead use statistics and learning examples to produce programs that either give better results than "hard-coded" solutions or provide good results where hard-coding a solution is virtually impossible. While there are

1 | https://portal.dbmi.hms.harvard.edu/projects/n2c2-nlp/ 


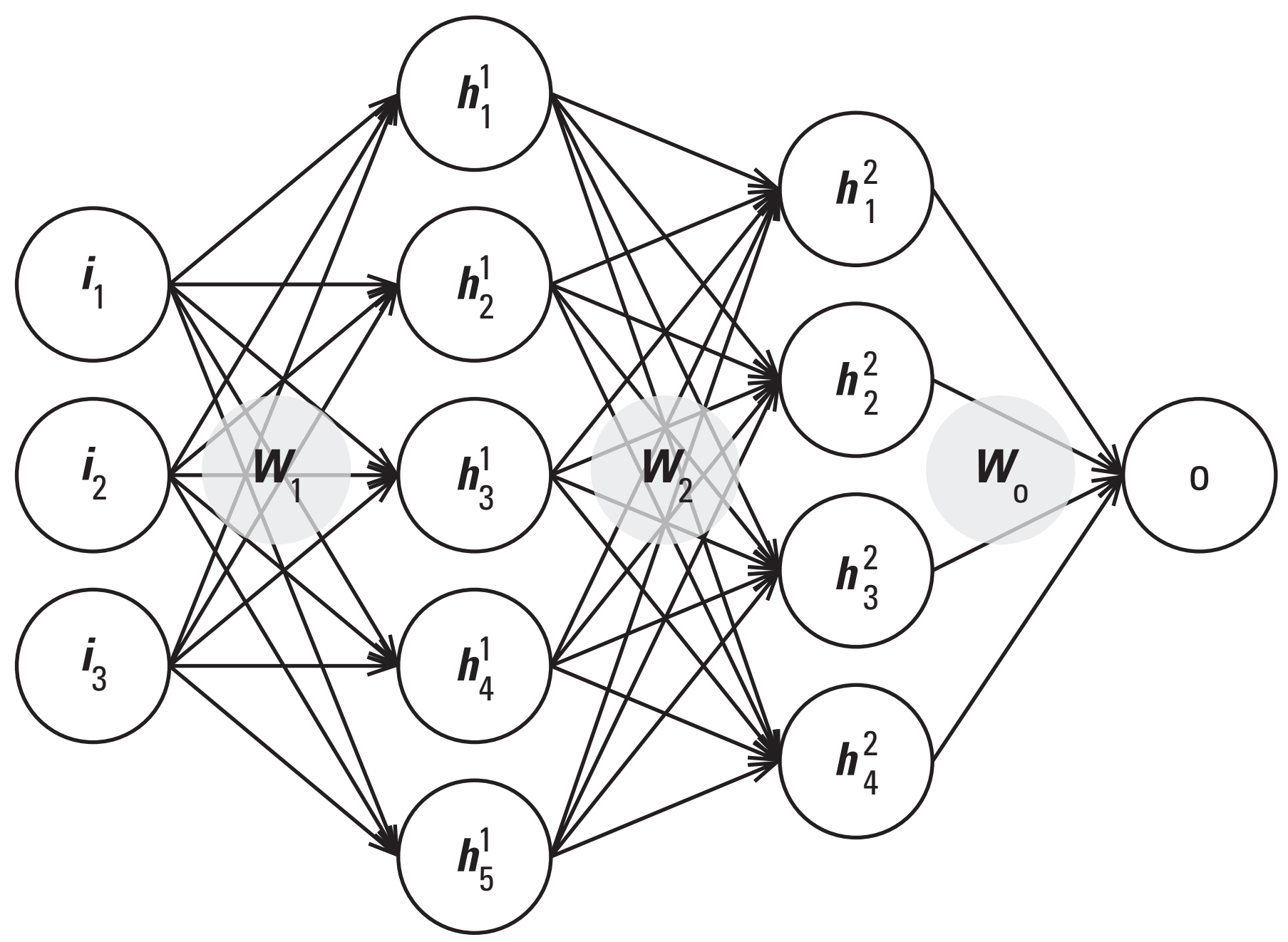

many different machine learning techniques known and successfully applied across a wide variety of problems, one specific approach - artificial neural networks (ANN) - has produced state-of-the-art results in recent years that render many previously go-to techniques obsolete. ANN is, in essence, a series of linear transforms (usually weighted sums) of inputs with usually very simply non-linear transforms (typically cutting off negative values, applying logistic functions, and similar) applied in between and a suitable interpretation of the output value(s) (e.g. class probabilities). An example of a simple neural network architecture is shown in Figure 1.

The input to the network that is depicted is a vector of dimension 3, called an input vector. The network projects the inputs by a matrix $\mathrm{W}_{1}$ to a vector of dimension 5 and applies a non-linearity to each of its components. The resulting vector $h^{1}$ is called a hidden representation, and the corresponding layer of neurons a hidden layer. The example network then projects $\mathrm{h}^{1}$ by $\mathrm{W}_{2}$ to a higher-order hidden representation $\mathrm{h}^{2}$, which is finally projected to the output by $\mathrm{W}_{3}$. This architecture with, e.g., a sigmoid function applied at the output layer, is suitable for binary classification. The network output is interpreted as a probability of inputs corresponding to one of the classes. The network "learns" from data by adjusting components of the projection matrices on the basis of the gradient of a loss function quantifying the discrepancy between the network output and the target "true" output.

\subsection{ANNs in natural language processing}

Natural language processing is an interdisciplinary field between linguistics and computer science focused on the interaction between a computer and a human through natural language (e.g. English). ANNs have gained traction in the field in recent years as a result of many separate improvements that came together to enable well-functioning models. Specifically, the advent of transformers (Vaswani et al., 2017) led to improvements in text-to-text tasks such as answering questions or, indeed, leading a conversation. The transformer architecture is shown in Figure 2. 
Figure 2 | Transformer architecture (taken from Vaswani et al., 2017)

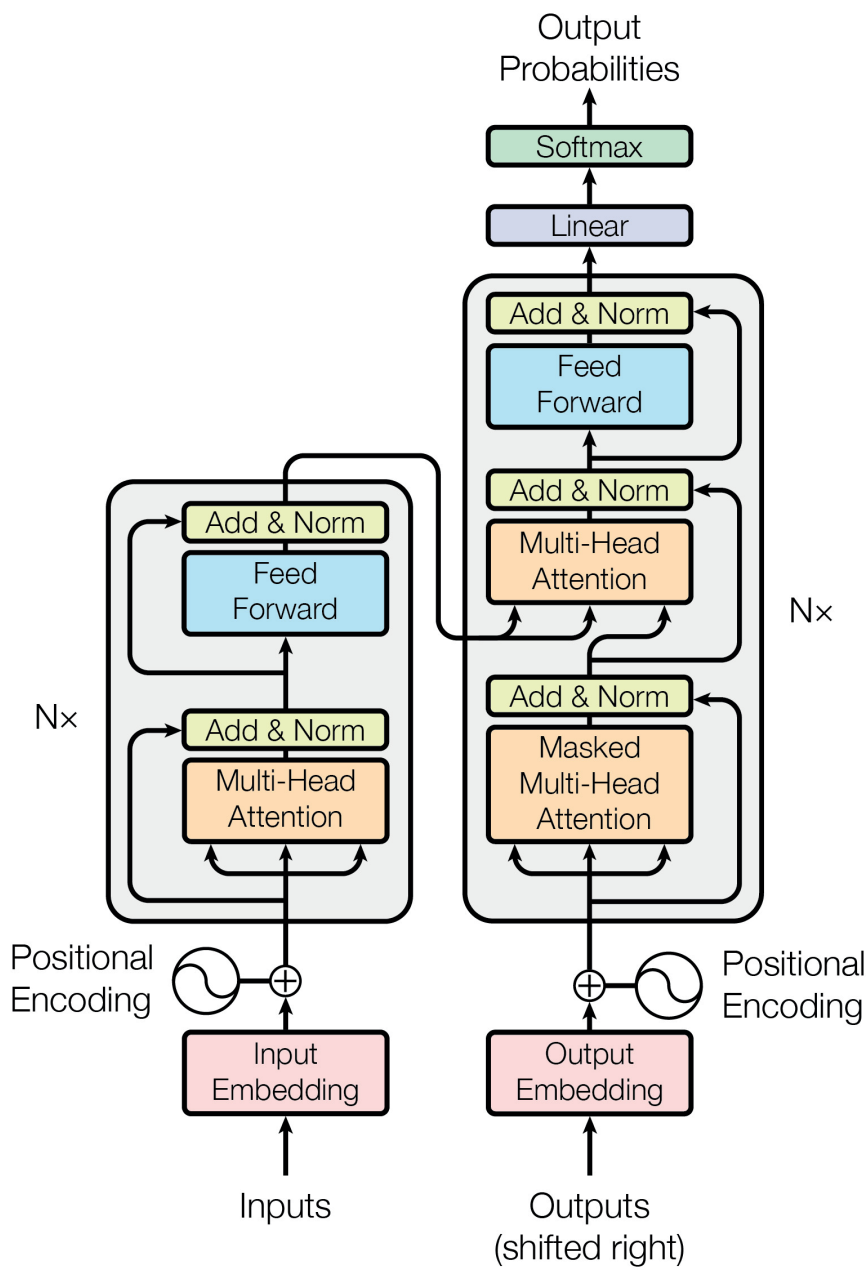

In the case of this architecture being employed to behave as a chatbot, it works roughly as follows. When the model is asked to reply to the last utterance of the counterparty, the whole conversation history is fed into the inputs shown on the left-hand side of the figure. Then the stack of layers on the left-hand side, called an encoder, produces highly abstracted hidden representations of every word in the conversation history. Each word representation is informed by all the other words from the history through an attention mechanism. The stack on the right-hand side is called a decoder. The reply is generated one word at a time. The generation depends on the encoded conversation history and the portion of the reply generated up to the given point. From these inputs, the decoder stack then produces scores for all vocabulary words and the highest-scoring word is selected as the following word in the reply. A full description is given in the original article (Vaswani et al., 2017).

The main obstacle to applying such models to a specific real-world problem is that they are significantly "data-hungry", while for any given problem, there might be barely any data at all. In the NLP domain, this can be, to some extent, alleviated by transfer learning. The core idea is to pre-train a model on tasks related to the target task, usually dubbed downstream task in the field, for which training data is abundant. This is especially amenable in NLP because of the vast amounts of textual data available from the World Wide Web. A recent comprehensive survey (Raffel et al., 2020) of transformer-based models able to process different text-to-text tasks combined with the release of code, well-performing pre-trained models, and a large ready-to-use "Colossal Clean Crawled Corpus" invites the application of NLP to new tasks.

\subsection{Informed addiction treatment conversational agent}

With the above-explained motivations for digital behavioural therapy, the limitations of the current "hard-coded" design approach, and recent relevant advances in NLP, an opportunity to create a transformer-based conversational agent presents itself. Since there is no readily available data to fine-tune the agent with for the smoking cessation domain, the proposed solution consists of two high-level steps. In the first step, the model will merely suggest replies to the expert in human-tohuman chat-based counselling, gradually making the task simpler for the professionals by saving the need to type. This would allow the system to learn on curated replies, or rather whole conversations containing the replies sanctioned by a trained professional. Once the model is sufficiently trained, tested, and approved, it could take the task over entirely and start helping on a larger scale.

\subsection{Human-to-human chat counselling}

The system will comprise the model itself, a chat interface for trained professionals, integration with messaging platforms (Signal) enabling communication at the user end, and integration between these components. The model itself is a pre-trained transformer ANN from the T5 family (Raffel et al., 2020), consuming the conversation history as input and returning an answer suggestion. To improve the results, the input may be extended with contextual information in plain text when appropriate. The addictology expert's chatting interface is a simple web application allowing logging in and out, managing sessions with smokers, and chatting itself. A part of the conversation history with a (somewhat irrelevant) suggested reply is shown in Figure 3. The integration with the messaging platforms needs to allow smokers to add counselling as a contact, view their availability, and chat with the agent. The integration between the components is chiefly responsible for managing the availability of counselling for smokers. The flow of the data is depicted in Figure 4. When the smoker is in a live session and sends a message (1), the integration relays the messages to the model (2), which provides a suggested reply (3) and subsequently delivers both the message and the suggested answer to the counsellor's interface (4). The counsellor then modifies the reply as they see fit and confirms. The reply is then relayed back to the smoker (6) but also back to the model for learning (5). 
Figure 3 | Chat interface with a piece of conversation and a suggested answer

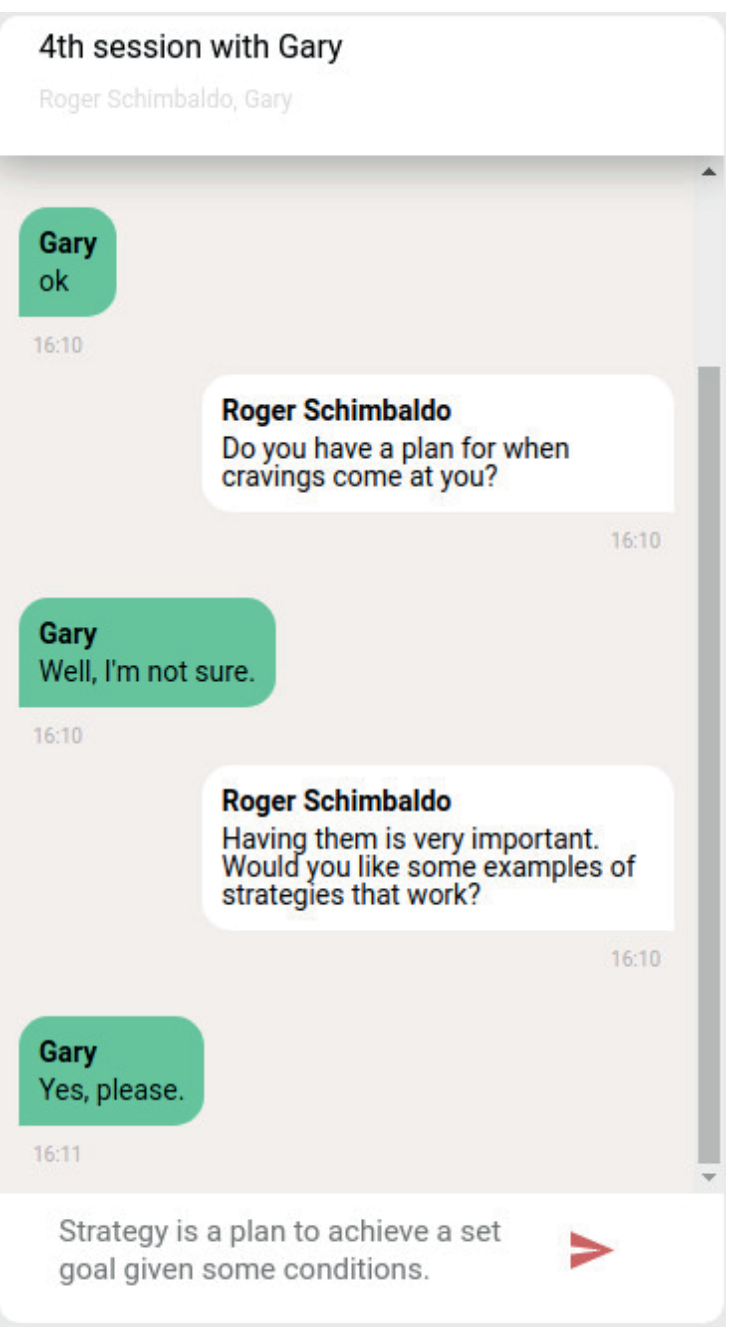

Figure 4 | Conversation data flow

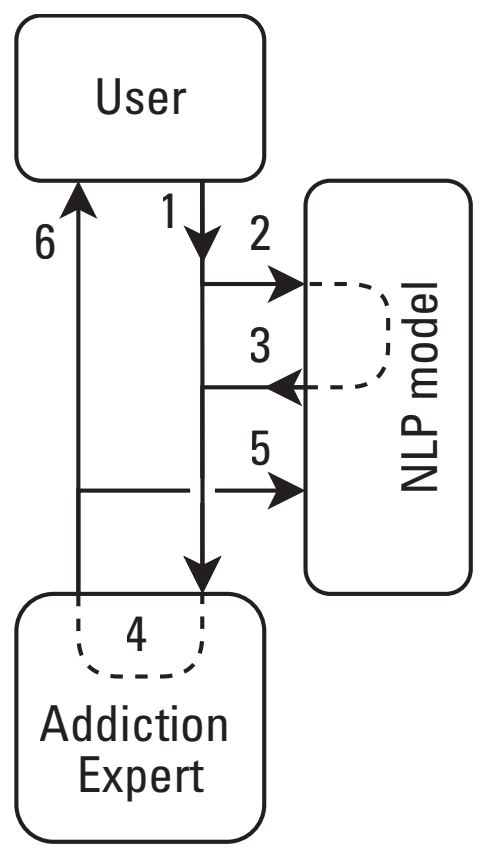

\subsection{Computer-to-human chat counselling}

From a technical perspective, the complete solution is slightly simplified when switching from the above computer-assisted human-to-human system to a computer-to-human one. Instead of presenting the suggestion to the counsellor, it simply sends the message suggested by the model back to the user. However, one has to consider that in the human-to-human setting, the model is kept on track by being fed a conversation history containing the "right" answers. This is similar to the teacher-forcing concept in machine learning, where the model is given correct answers to previous questions to produce the next answer. If not controlled for, this could mean that the conversation would gradually stray off the intended path. Therefore, the system needs the ability to detect that the conversation has moved away from the topic. Once the system detects this has occurred, it can apologise for its own confusion and then offer to start over either from the beginning of the session or a checkpoint possibly picked by the user.

\subsection{Comparison to previous work}

The approach presented here is largely motivated by an eHealth smoking cessation application described in Kulhánek et al. (2018). The technical core of this application is a predefined decision tree with users traversing the tree according to their situation. The therapeutic fundament of this aid lies in cognitive-behavioural therapy with a focus on motivational interviews, positive reinforcement, lapse preparation, and lapse management. The work described here aims to replace the decision tree with a T5-based chatbot and plain-text domain knowledge while using the same therapeutic approach.

To my knowledge, the only previously suggested chatbot-based aid for smokers trying to quit is the one described in Calvaresi et al. (2019). Their study showed high efficacy; $28.9 \%$ of the participants did not smoke three months after quitting. The approach suggested in this article differs in two ways. Firstly, the chatbot model itself is fundamentally different; the chatbot used by Calvaresi et al. (2019) is a state machine, in principle close to the decision tree approach and therefore necessarily somewhat rigid in the interactions compared to a good ANNbased agent. The second difference is the therapeutic base. Although both programmes consist of a pre-quitting and a post-quitting phase, the former actively prepares the smoker for quitting through cognitive-behavioural therapy methods, while the latter merely collects the data, such as the number of cigarettes per day or the user's smoking habits, to reuse it in the post-quitting phase and to present it concisely to the user to increase their motivation. The ANN-based agent should learn to mimic a trained addiction expert in providing comprehensive cognitive-behavioural cessation therapy.

\section{DISCUSSION}

The proposed conversational agent would offer internetdelivered cognitive behavioural therapy for smoking cessation available to a substantial number of smokers worldwide. As 
a result of increased engagement, the hope is for the agent's therapy efficacy to be higher than that of the previously developed systems described above. By its nature, managing its contents would be less costly in terms of addiction expert time compared to a rule-based system. Furthermore, once the system is operable in one language, it is easily extendable to other languages. This is highly beneficial because of the vast number of smokers in non-English-speaking countries (Gowing et al., 2015). If it were successful, the same framework could be applied to other tasks, e.g. alcohol abuse therapy delivery.

The most significant advantage of the system described here, as compared to conventional therapy, is its availability in two regards. It is able to accommodate many users. From the user's perspective, the ability to follow the therapy at a convenient time without travelling to the counsellor's practice or accessing it when acutely needed is highly beneficial.

Notwithstanding the benefits described above, the suggested approach is not without problems. Firstly, despite recent promising results in transfer learning of transformers, creating an agent as described of sufficient quality might prove just beyond the reach of the current technical possibilities. Secondly, collecting the sensitive data required for training the system is not straightforward. Precautions have to be taken to ensure no personal data is retained either in stored examples or in the ANN itself. These precautions need to be deemed sufficient by the responsible ethical committees, and at the same time, they must not limit the learning of the model too much. Without solutions to these two basic problems, the model described here cannot come into existence.

The ethical aspects remain important even in the second stage, where de-identifying data is not possible before a given user finishes the programme as their therapy history is required for continuation. While this poses an issue, robust technical solutions, e.g. per-user therapy history encryption, are available. Sharing de-identified data is both desirable to facilitate research in the fields that are affected and problematic from the ethical perspective, as pointed out by Velupillai et al. (2018). However, if these issues are solved, the system could support research in the tobacco addiction field, allow the verification of hypotheses, or enable the identification of weak spots of the treatment.

From the client's perspective, there are advantages and drawbacks to consuming a smoking cessation therapy delivered by an automated conversational agent compared to the conventional in-practice therapy. A subset of both stems from the presence or lack of presence of the counsellor. The counsellor can identify issues in the therapy early and e.g. suggest a different treatment. Sometimes, they can offer a complementary medication or use therapeutic techniques beyond the possibilities of text-based chat. On the other hand, the programmed agent will always follow the set therapy, while the counsellors can, at times, drift away from the therapy manual. As already mentioned above, a great advantage of the electronic version of the therapy is its convenience for the user. Further, if designed well, the system can offer the possibility of revisiting topics already covered on demand, depending on what a given user would like to remind themselves of at any time.

\section{CONCLUSION}

A case has been made for a transformer-like artificial neural network-based smoking cessation chatbot. Once functioning, such a system would be comparatively cheap to run and available at any time. Because of the lack of data for such models to learn from, a two-stage approach is suggested. The first stage involves addiction experts providing human-tohuman counselling via a chat interface, thus facilitating data collection. At the same time, they would also preview how the system is improving on the basis of the previous conversations with each system-suggested answer. This should ultimately enable the second stage, in which a fully autonomous smoking cessation behavioural therapy chatbot will be created. Based on the evidence of previous results from RCT (Kulhánek et al., 2021), a user acceptance study (Kulhánek et al., 2018), and other results, having such a system appears to be highly desirable. New techniques in natural language processing, along with making the relevant code, models, and data readily available (Raffel et al., 2020), make this endeavour feasible in the technical realm. A possible downside to using end-to-end trained ANN as a core model is a lack of ability to explain its behaviour. There is a risk of the model not behaving as desired without any clear paths to alleviate the problems. The system described here is currently a work in progress. 


\section{REFERENCES}

Abd-alrazaq, A. A., Alajlani, M., Alalwan, A. A., Bewick, B. M., Gardner, P., \& Househ, M. (2019). An overview of the features of chatbots in mental health: A scoping review. International Journal of Medical Informatics, 132. https://doi.org/10.1016/j.ijmedinf.2019.103978

Auriacombe, M., Moriceau, S., Serre, F., Denis, C., Micoulaud-Franchi, J. A., de Sevin, E., Bonhomme, E., Bioulac, S., Fatseas, M., \& Philip, P. (2018).

Development and validation of a virtual agent to screen tobacco and alcohol use disorders. Drug and Alcohol Dependence, 193, 1-6. https://doi.org/10.1016/j.drugalcdep.2018.08.025

Calvaresi, D., Calbimonte, J. P., Dubosson, F., Najjar, A., \& Schumacher, M. (2019). Social network chatbots for smoking cessation: Agent and multi-agent frameworks. Proceedings - 2019 IEEE/WIC/ACM International Conference on Web Intelligence, WI 2019, 286-292. https://doi.org/10.1145/3350546.3352532

Carrell, D. S., Cronkite, D., Palmer, R. E., Saunders, K., Gross, D. E., Masters, E. T., Hylan, T. R., \& Von Korff, M. (2015). Using natural language processing to identify problem usage of prescription opioids. International Journal of Medical Informatics, 84(12), 1057-1064. https://doi.org/10.1016/j.ijmedinf.2015.09.002

Conway, M., Hu, M., \& Chapman, W. W. (2019). Recent advances in using natural language processing to address public health research questions using social media and consumer-generated data. Yearbook of Medical Informatics, 28(1), 208-217. https://doi.org/10.1055/s-0039-1677918

Filannino, M., Stubbs, A., \& Uzuner, Ö. (2017). Symptom severity prediction from neuropsychiatric clinical records: Overview of 2016 CEGS N-GRID shared tasks Track 2. Journal of Biomedical Informatics, 75, S62-S70. https://doi.org/10.1016/j.jbi.2017.04.017

Gowing, L. R., Ali, R. L., Allsop, S., Marsden, J., Turf, E. E., West, R., \& Witton, J. (2015). Global statistics on addictive behaviours: 2014 status report. Addiction, 110(6), 904-919. https://doi.org/10.1111/add.12899

Jha, P., \& Peto, R. (2014). Global effects of smoking, of quitting, and of taxing tobacco. New England Journal of Medicine, 370(1), 60-68. https://doi.org/10.1056/nejmra1308383

Kulhánek, A., Gabrhelík, R., Novák, D., Burda, V., \& Brendryen, H. (2018). eHealth intervention for smoking cessation for Czech tobacco smokers: Pilot study of user acceptance. Adiktologie, 18(2), 81-85.

Kulhánek, A., Lukavská, K., Gabrhelík, R., Prokop, J., Burda, V., Novák, D., Holter, M. T. S., \& Brendryen, H. (2021). eHealth smoking cessation intervention in less adherent smokers: A randomized controlled trial comparing text messages and e-mail reminders. Manuscript submitted for publication.

McCowan, I., Moore, D., \& Fry, M. J. (2006). Classification of cancer stage from free-text histology reports. Annual International Conference of the IEEE Engineering in Medicine and Biology - Proceedings, 5153-5156. https://doi.org/10.1109/IEMBS.2006.259563

Raffel, C., Shazeer, N., Roberts, A., Lee, K., Narang, S., Matena, M., Zhou, Y., Peter, W. L., \& Liu, J. (2020). Exploring the limits of transfer learning with a unified text-to-text transformer. Journal of Machine Learning Research, 21(140), 1-67.

Roberts, A. (2017). Language, structure, and reuse in the electronic health record. AMA Journal of Ethics, 19(3), 281-288. www.amajournalofethics.org

Savova, G. K., Masanz, J. J., Ogren, P. V., Zheng, J., Sohn, S., Kipper-Schuler, K. C., \& Chute, C. G. (2010). Mayo clinical Text Analysis and Knowledge Extraction System (cTAKES): Architecture, component evaluation and applications. Journal of the American Medical Informatics Association, 17(5), 507-513. https://doi.org/10.1136/jamia.2009.001560

Vaswani, A., Brain, G., Shazeer, N., Parmar, N., Uszkoreit, J., Jones, L., Gomez, A. N., Kaiser, Ł., \& Polosukhin, I. (2017). Attention is all you need. Advances in Neural Information Processing Systems, Nips, 5998-6008. http://papers.nips.cc/paper/7181-attention-is-all-you-need.pdf
Velupillai, S., Suominen, H., Liakata, M., Roberts, A., Shah, A. D., Morley, K., Osborn, D., Hayes, J., Stewart, R., Downs, J., Chapman, W., \& Dutta, R. (2018) Using clinical Natural Language Processing for health outcomes research: Overview and actionable suggestions for future advances. Journal of Biomedical Informatics, 88, 11-19. https://doi.org/10.1016/j.jbi.2018.10.005

Wang, Y., Wang, L., Rastegar-Mojarad, M., Moon, S., Shen, F., Afzal, N., Liu, S., Zeng, Y., Mehrabi, S., Sohn, S., \& Liu, H. (2018). Clinical information extraction applications: A literature review. Journal of Biomedical Informatics, 77, 34-49. https://doi.org/10.1016/j.jbi.2017.11.011

West, R. (2017). Tobacco smoking: Health impact, prevalence, correlates and interventions. Psychology and Health, 32(8), 1018-1036. https://doi.org/10.1080/08870446.2017.1325890

Wu, C. S., Madotto, A., Hosseini-Asl, E., Xiong, C., Socher, R., \& Fung, P. (2020). Transferable multi-domain state generator for task-oriented dialogue systems. ACL 2019 - 57th Annual Meeting of the Association for Computational Linguistics, Proceedings of the Conference, 808-819. 